\title{
CALCULATIONS OF IMPIJRITY RADIATION AND ITS \\ EFFECTS ON TOKAMAK EXPERIMENTS
}

BY

$R, V$, JENSEN AND D. E. POST W. H. GRASBERGER, C. B. TARTER, AND

W. A. LOKKE

\section{PLASMA PHYSICS LABORATORY}

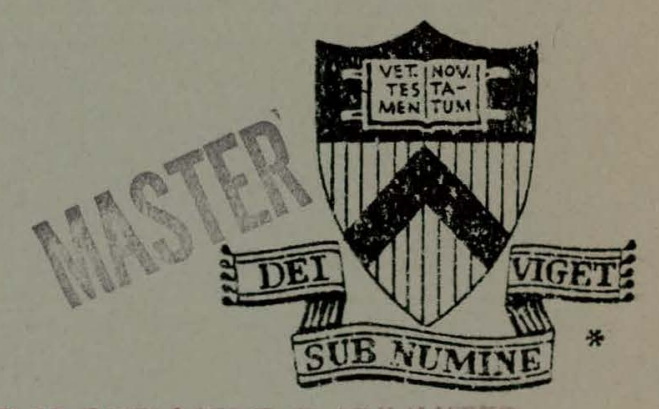

DISTRIBUTION OF THIS DQCUMENT IS UNEIMITED

\section{PRINCETON \\ UNIVERSITY PRINCETON, NEW JERSEY}

This work was supported by U. S. Tnergy Research and Development Administration Contract $\mathrm{E}(11-1)-3073$. Reproduction, translation, publication, use and disposal, in whoie or in part, by or for the united states Governnent is pernitted. 


\section{DISCLAIMER}

This report was prepared as an account of work sponsored by an agency of the United States Government. Neither the United States Government nor any agency Thereof, nor any of their employees, makes any warranty, express or implied, or assumes any legal liability or responsibility for the accuracy, completeness, or usefulness of any information, apparatus, product, or process disclosed, or represents that its use would not infringe privately owned rights. Reference herein to any specific commercial product, process, or service by trade name, trademark, manufacturer, or otherwise does not necessarily constitute or imply its endorsement, recommendation, or favoring by the United States Government or any agency thereof. The views and opinions of authors expressed herein do not necessarily state or reflect those of the United States Government or any agency thereof. 


\section{DISCLAIMER}

Portions of this document may be illegible in electronic image products. Images are produced from the best available original document. 
NOTICE

This report was prepared as an account of work sponsored by the United States Government. Neither the United States nor the United States Energy Research and Development Administration, nor any of their employees, nor any of their contractors, subcontractors, or their employees, makes any warranty, express

or implied, or assumes any legal liability or responsibility for the accuracy, completeness or usefulness of any information, apparatus, product or process disclosed, or represents that its use would not infringe privately owned rights.

Printed in the United States of America.

$$
\text { Available from }
$$

National Technical Information Service

U. S. Department of Commerce 5285 Port Royal Road Springfield, Virginia 22151

Price: Printed Copy $\${ }_{+}$: Microfiche $\$ 3.00$

\begin{tabular}{lr} 
*Pages & \multicolumn{1}{c}{$\begin{array}{r}\text { Sel1ing Pr } \\
1-50\end{array}$} \\
$51-150$ & $\$ 4.00$ \\
$151-325$ & 5.45 \\
$326-500$ & 7.60 \\
$501-1000$ & 10.60 \\
& 13.60
\end{tabular}


Calculations of Impurity Radiation and Its

Effects on Tokamak Experiments*

\author{
R. V. Jensen and D. E. Post \\ Flasma Thysics Laboratory \\ Princeton University \\ Princeton, New Jersey 08540
}

and

W. H. Grasberger, C. B. Tarter and W. A. Lokke

Lawrence Livermore Laboratory

Livermore, California 94550
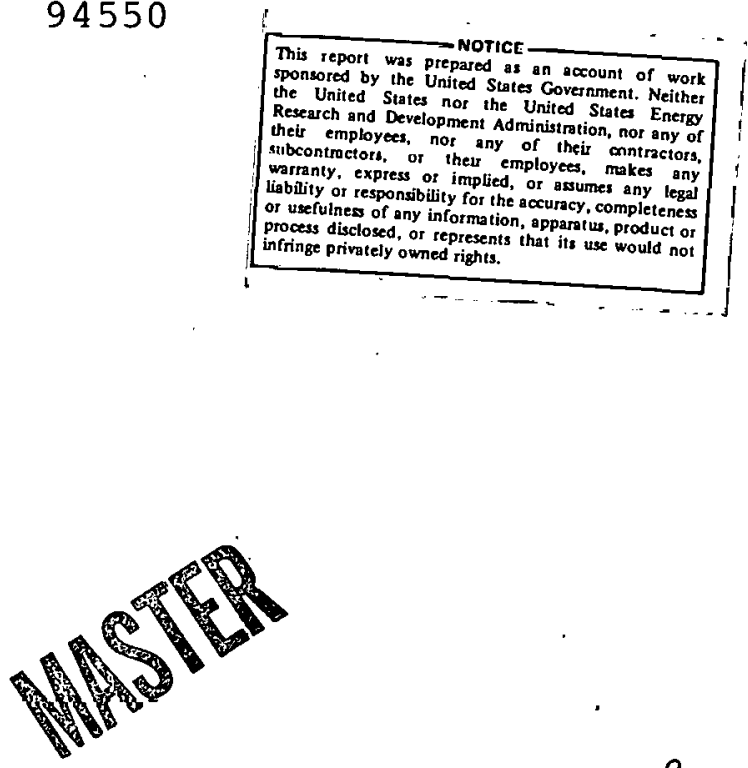


\section{ABSTRACT}

The impurity radiation for typical tokamak parameters has been numerically calculated using an "average-ion model." Coronal equilibrium values for the emission of oxygen, iron, molybdenum, tungsten and gold were determined from the steady-state solutions of a set of related rate equations which included the effects of electron collisional ionization and excitation, dielectronic and radiative recombination, $\Delta \mathrm{n}=0$ and $\Delta \mathrm{n} \neq 0$ line transtions, and bremsstrahlung. The results for oxygen and iron compare very well with other calculations. However, for $\mathrm{T} \leq 10 \mathrm{keV}$ the high-Z impurity radiation is significantly higher than previous estimates. Since impurities diffusing in a tokamak are not expected to be in coronal equilibrium, time-dependent radiation calculations were also performed. ' $\Lambda$ comparison of these nonequilibrium loss rates with those calculated under the assumption of coronal equilibrium indicates that coronal radiation calculations do not significantly underestimate the moderateand high-z impurity radiation losses for neoclassical diffusion velocities in large tokamaks, such as PLT(Princeton Large Torus) and TFTR(Tokamak Fusion Test Reactor) . Finally, the detailed steady-state emission rates were used to investigate the effects of various concentrations of impurities on the $\mathrm{n}_{\mathrm{e}}^{\tau}$ requirements for breakeven, ignition, and $Q=5$ beam driven reactor experiments. 


\section{INTRODUCTION}

The significance of impurity radiation in tokamak plasmas has been well established. Impurity radiation accounts for a large fraction of the energy loss from TFR (Tokamak Fontenayaux-Roses) and other tokamaks. [1,2]. Moreover, radiation from high-z impurities such as molybdenum, tungsten and gold is expected to be one of the limiting factors in the operation of large tokamaks. $[3,4]$.

Unfortunately, due to the uncertalntles dud cuniplexities of the atomic physics, the detailed calculation of impurity radiation losses has been limited to low- and moderate-z elements [5, $6,7,8,9]$. For $z>26$ previous work consists primarily of order of magnitude estimates such as:

$$
P_{z}=\left(10^{-25} \rightarrow 10^{-26}\right) \mathrm{n}_{e^{n}} \text { watts } / \mathrm{cm}^{3}
$$

given by Hinnov [10] and calculations for highly stripped impurity ions at thermonuclear temperatures $(\mathrm{T} \geq 10 \mathrm{keV})$ [11]. However, these estimates generally neglect some of the dominant factors contributing to impurity radiation.

We have performed detailed time dependent calculations of the radiative losses for elements ranging from hydrogen $(Z=1)$ to gold $(\mathrm{z}=79)$ for tokamak plasma temperatures between $2 \mathrm{eV}$ and $100 \mathrm{keV}$. Our zero-dimensional numerical calculation uses an "average ion model" which permits in principle the simple treatment of impurities with arbitrary $\mathrm{z}$. The calculation includes: 
1) electron collisional ionization and excitation and their inverse processes; 2) dielectronic and radiative recombination; 3) $\Delta \mathrm{n} \neq 0$ and $\Delta \mathrm{n}=0$ radiative line transitions; and 4) bremsstrahlung. The rate coefficients for some of these atomic processes are frequently uncertain by factors of 2 to 4 . Consequently, simplifications in the calculation of impurity radiation, such as the average ion model, seem justified at present.

By running our code at constant temperatures and densities to a steady state, we have calculated "coronal equilibrium" values for the radiated power, $\overline{\mathrm{z}}$ and $\overline{\mathrm{z}^{2}}$ for impurities in a tenuous hydrogen plasma. The results for low- and moderate-z elements compare very well with previous work, and the high-z impurity radiation is within a factor of 1.5 of earlier results [11] for $\mathrm{T}>20 \mathrm{keV}$.

In general previous estimates of high-z impurity radiation have neglected the effects of dielectronic recombination and have employed hydrogenic oscillator strengths. In steady-state calculations, however, we find that dielectronic recombination is the dominant recombination process. We also find that the inclusion of $\Delta \mathrm{n}=0$ transitions, and the use of relativistic HartreeFock oscillator strengths for high-z elements can be quite important. As a result our calculations for high-z impurity radiation turn out to be significantly higher than previous estimates for $\mathrm{T}_{\mathrm{e}} \leq 10 \mathrm{keV}$. In particular, for ohmic-heated tokamak 
temperatures $(T \sim l \rightarrow 2 \mathrm{keV})$, the emission rates for tungsten and gold are $\sim 6 \rightarrow 60$ times greater than those predicted by Eq. (1). In addition to steady-state calculations, time dependent temperature and density sources were used in our calculation to assess the accuracy of the assumption of coronal equilibrium in tokamaks such as ATC (Adiabatic Toroidal Compressor), PLT (Princeton Large Torus), and TFTR (Tokamak Fusion Test Reactor) for the determination of impurity radiation loss rates. Fur sufficienlly rapid diffusion velocities and steep temperature gradients, we find coronal equilibrium to be a poor assumption. However for large tokamaks with neoclassical inward diffusion velocities, coronal equilibrium appears to provide reasonable upper limits for moderate- and high- $\mathrm{z}$ radiation losses.

Finally, using our radiation calculations we have investigated the effects of impurity radiation losses on fusion reactor experiments. In addition to Lawson and ignition criteria, we have calculated the $\mathrm{n}^{\tau}$ requirements for a $Q=5$ ( $Q=$ total fusion energy/beam energy) neutral deuteron beam-driven reactor as functions of the impurity level. These results clearly demonstrate the dramatic effects of small concentrations of high-z impurities on the energy balance. For a lokeV D-T plasma, tungsten concentrations of $0.052 \%, 0.03 \%$, and $0.012 \%$ of the hydrogen ion density are sufficient to prevent breakeven, $Q=5$, and ignition experiments respectively. 
II. AVERAGE ION MODEL

Tokamak plasma impurities appear in a number of ionization states. An exact calculation of impurity radiation requires the treatment of each ionic species. A useful approximation, which has been successfully employed in the calculation of high-z opacities, is to replace the different ionic species for each element with a single fictitious "average ion." In the average ion model, the presence of the various ionization states in a real plasma is statistically accounted for by fractional electron populations in the principal electron shells of the average ion with $\mathrm{z}_{\text {av }}=\overline{\mathrm{z}}$. This simplification was first utilized by Strömgren $[13,14]$ in 1932 to calculate the bound-free contribution to stellar opacities.

As an illustration of this approach, consider a $10^{14}$ electrons $/ \mathrm{cm}^{3}$ hydrogen plasma with $10^{12}$ iron ions $/ \mathrm{cm}^{3}$ at $1.5 \mathrm{keV}$. The electron populations for the principal shells $n=1,2,3 \& 4$ of the average iron ion are:

$\mathrm{F}_{1}=2.000 \quad \mathrm{~F}_{2}=1.099 \quad \mathrm{r}_{3}=2.897 \times 10^{-10} \quad \mathrm{H}_{4}=1.486 \times 10^{-1.0}$

The corresponding ionic species densities $\mathrm{n}_{i}$ is shown in Table 1 . In this example, the $K$ and $L$ shell electron populations of the average ion are just equal to the weighted sum of the contributions of each of the ionization states. The populations of the higher levels represent excited electrons.

The state of the average ion plasma is completely determined by the specification of the electron level populations for each 
element. The time evolution of the state of the plasma can, therefore, be calculated by solving rate equations for these populations:

$$
\begin{gathered}
\frac{d P_{n}}{d t}=\left[Q_{n} \cdot\left(R_{n}+\sum_{m>n} D L_{n, m} \cdot P_{m}+\sum_{m<n} U L_{n, m} \cdot P_{m}\right)+D_{n}\right] \\
{\left[\mathrm{I}_{n} \cdot\left(C_{n}+\sum_{m, n} U L_{m, n} \cdot Q_{m}+\sum_{111} D L_{m, n} \cdot \Omega_{m}\right)\right]}
\end{gathered}
$$

where $Q_{n}=\left(2 n^{2}-P_{n}\right)$ is the number of holes in the $n^{\text {th }}$ shell

$$
\begin{aligned}
\mathrm{R}_{\mathrm{n}} & =\text { Radiative recombination rate } \\
\mathrm{DL}_{\mathrm{n}, \mathrm{m}} & =\text { Downward line transition rate } \mathrm{m} \rightarrow \mathrm{n} \\
\mathrm{UL}_{\mathrm{n}, \mathrm{m}} & =\text { Upward line transition rate } \mathrm{m} \rightarrow \mathrm{n} \\
\mathrm{C}_{\mathrm{n}} & =\text { Collisional ionization rate } \\
\mathrm{D}_{\mathrm{n}} & =\text { Excitation rate to the } \mathrm{n}^{\text {th }} \text { shell due to Dielectronic } \\
& \text { Recombination. }
\end{aligned}
$$

In our calculation, these coupled nonlinear equations are solved numerically using a Newton-Rapheson method. At each time step, the resulting electron populations are used to calculate the average ion energy levels using a Bohr model with screening factors and relativistic corrections. Finally, with these average ion energy levels, populations and corresponding transition rates, the emission due to bremsstrahlung, radiative and dielectronic recombination, and line transitions is calculated. 
The rates for radiative recombination, collisional excitation, and collisional ionization for various ionic species differ primarily in the values for the principal shell populations and energy levels. Since the energy levels of adjacent ionization states are not significantly different and the total rates are approximately linear in the numbers of principal shell electrons, the average ion model is adequate for the calculation of these contributions to the impurity radiation.

In addition the impurity bremsstrahlung is also accurately calculated in the average ion approximation since it depends upon $\overline{z^{2}} \sim \bar{z}^{2}=z^{2}$

Ion

However, dielectronic recombination and $\Delta \mathrm{n}=0$ transition rates are more sensitive to the specific ionization states. Although exact knowledge of the ionization states was sacrificed in adopting the average ion model, approximate ionic species densities are recovered for the calculation of these processes by specifying ratios between adjacent ionic species and by requiring that:

$$
P_{m}=\sum_{i} n_{i} P_{m}(i), \quad \sum_{i} n_{i}=1
$$

where $n_{i}$ is the fractional density of the $i^{\text {th }}$ ionization state and $\mathrm{P}_{\mathrm{m}}(i)$ is the $\mathrm{m}$ shell population of the $i^{\text {th }}$ state. In steady state, the density ratios are determined by coronal equilibrium:

$$
n_{i} / n_{i+1}=R_{i+1} / C_{i}
$$


where $R_{i+1}$ is the recombination rate of the $i+1$ species and $c_{i}$ is the collisional ionization rate of the $i^{\text {th }}$ species. For. time dependent problems, the right-hand side of Eq. (4) is multiplied by an additional unknown variable $x(t)$ which is determined from Eqs. (3) and (4) which give $N+1$ equations for $N$ unknown species densities. Since Eq. (4) emphases steady-state ratios, this prescription is most accurate for systems close to steady state. Despite the approximations involved in the averageion model, comparisons with other calculations using fully ionic species codes indicate the model is well within the uncertainties of the atomic physics. (See Table 1 and Figs. 1-2)

III. ATOMIC PHYSICS

For the purposes of comparison with other radiation calculations, the sources for the atomic physics data used in our calculation are reviewed below:

1. Collisional Ionization

The ionization rate is similar to Jordan's.[15] with a modified gaunt factor. The gaunt factor results from polynomial fits to more detailed ionization rates [16].

The inverse process - three body recombination - is determined by the principle of detailed balance.

\section{Collisional Excitation}

The excitation rate is given by Bates, et.al. [17] with a polynomial gaunt factor. The oscillator strengths were determined by relativistic Hartree-Fock calculations for $\Delta \mathrm{n}=1$ 
transitions [12] their hydrogenic values for $\Delta \mathrm{n} \geq 2$, and by fits to calculated and observed, values for $\Delta n=0[12,16]$.

The collisional de-excitation rate is again calculated from detailed balance.

\section{Dielectronic Recombination}

We use Burgess' $[18,19]$ recombination rate with a high density correction [16] and an additional reduction factor for $\Delta \mathrm{n} \neq 0$ transitions suggested by Mercs et.al. [7].

Dielectronic recombination occurs when a free electron is captured in a high-lying autoionizing level by exciting a bound electron. It is an important process in tokamak plasmas because the excited and captured electrons decay radiatively before collisional ionization occurs. We assume that the captured electron cascades immediately to the ground state. The radiation due to dielectronic recombination is, therefore, equal to the ionization potential of the ground state of the ion after recombination. The additional radiation from the decay of the excited electron is simply added to the line radiation.

4. Radiative Recombination

The recombination rate is the lowest order expression of Seaton [20]. The recombination radiation is summed over the principal shells of the average ion for the total bound-free radiation.

\section{Line Radiation}

The $\Delta \mathrm{n} \neq 0$ line radiation for the average ion is determined by the spontaneous transition rates [21] between the principal electron levels in the average ion. 
The $\Delta \mathrm{n}=0$ line radiation is calculated for each ionic species using polynomial fits to detailed calculations of relativistic energy levels and oscillator strengths [16]. The ionic species densities are determined in the average ion model by the procedure described in Sec. II.

\section{Bremsstrahlung}

The free-free emission rate is given by the classical expression using the gaunt factor of Karzas and Latter [22].

\section{STEADY-STATE CALCULATIONS}

The steady-state (coronal) radiation has been calculated as a function of electron temperature for a variety of impurities in a transparent hydrogen plasma. Comparisons with previous calculations for oxgyen and iron are shown in Figs. 1 and 2. Because of the versatility of the average-ion model calculations for higher $\mathrm{z}$ elements were possible, Figs. $3-5$ show the normalized emission rates for molybdenum, tungsten and gold. The radiation rates for these impurities are of great interest to the fusion community because of their use as tokamak wall and limiter materials.

The increase in impurity radiation with $\mathrm{Z}$ is illustrated in Fig. 6. These curves emphasize the importance of the high-z impurities. 


\section{COMPARISON OF EQUILIBRIUM AND NON-EQUILIBRIUM IMPURITY RADIATION}

Impurity ions diffusing in a plasma are not expected to be in coronal equilibrium. The effects of tokamak diffusion are simulated by using estimates for inward diffusion velocities to transform radial temperature and density profiles into time dependent sources. With these temperature and density sources, we calculate the non-equilibrium ionization states and radiation of a Lagrangian volume element of diffusing impurities. Our code is then run to steady state at various temperatures and densities corresponding to particular times (or radial positions) to determine the coronal radiation and $\bar{z}$. A comparison of these results gives an estimate for the errors in coronal calculations of impurity energy losses in tokamaks.

Such calculations have been performed for moderate- $\mathrm{Z}$ impurities in ATC (Adiabatic Toroidal Compressor), PLT (Princeton Large Torus) and TFTR (Tokamak Fusion Test Reactor) with experimental and theoretical temperature and density profiles and with the assumption of neoclassical (Pfirsh-Schlüter) values for inward diffusion velocities $\left(v_{\max } \sim 0.5-2 . \mathrm{cm} / \mathrm{ms}\right)$. The parameters for these calculations are listed in Table 2 .

These comparisons indicate that for transport code calculations for small devices such as ATC (Adiabatic Toroidal Compressor) with large gradients and high diffusion velocities coronal equilibriur is a poor approximation. However, under the assumption of 
Pfirsh-Schlüter inward diffusion velocities for moderate $\mathrm{Z}$ impurities in large tokamaks such as PLT (Princeton Large Torus) and TFTR (Tokamak Fusion Test Reactor) coronal equilibrium does not significantly underestimate the total impurity energy loss since the bulk of the radiation comes from the interior of the plasma where the gradients are relatively flat.

Unfortunately, because of uncertainties in the impurity transport, the accuracy of the assumption of coronal equilibrium remains an open question. Inward diffusion velocities much greater than neoclassical will increase the disparity between equilibrium and non-equilibrium impurity radiation. In addition coronal equilibrium calculations may be expected to overestimate the contributions due to outward diffusing ions.

\section{1) ATC (Adiabatic Toroidal Compressor)}

Using the temperature, density and velocity profiles from the experiments of Marmar, Cohen and Cecchi [23], we simulated the diffusion of injected aluminum ions in ATC. Figures 7a and $7 \mathrm{~b}$ show the difference between the equilibrium and nonequilibrium calculations for $\bar{z}$ and radiated power. The coronal calculation strips out the aluminum ions much faster. This difference results in a large disparity in the magnitudes of the radiated energy.

2) PLT (Princeton Large Torus)

For simplicity, a neoclassical radial diffusion velocity was estimated by the following formula [24]:

$$
v(r)=2 \cdot \frac{8(2 \pi)^{1 / 2}}{3} \frac{e^{2} c^{2} m_{H}^{1 / 2}}{B_{z}^{2}\left(k T_{i}\right)^{1 / 2}} \ln \Lambda\left(1+q^{2}\right) \bar{z} \frac{d n_{H}}{d r}
$$


where $\mathrm{B}_{z}$ is the magnitude of the toroidal field, $T_{i}$ is the ion temperature and $q^{2}=\left[1+2(r / a)^{2}\right]^{2}, \quad(a=$ minor radius $)$.

Experience indicated that the greatest disparity between coronal and non-coronal calculations could be expected near the edge where the gradients and velocities are largest. The diffusion velocities were, therefore, chosen to have constant values greater than or equal to the neoclassical velocity at the edge. This would be expected to give an upper bound on the time dependent effects.

In Figs. $8 \mathrm{a}$ and $8 \mathrm{~b}$ the comparisons of $\overline{\mathrm{z}}$ and radiated power for iron diffusing inward in PLT (Princton Large Torus) at velocities of $0.5 \mathrm{~cm} / \mathrm{ms}$ and $2 . \mathrm{cm} / \mathrm{ms}$ are shown [Eq. (5) gives $\left.\mathrm{v}_{\text {edge }} \cong 0.42 \mathrm{~cm} / \mathrm{ms}\right]$. Figure $8 \mathrm{c}$ shows similar results for molybdenum. The simulations were terminated at distances of 20 $\mathrm{cm}$ and $9 \mathrm{~cm}$ respectively as the differences between the calculations were no longer noticeable.

It is apparent from these results that, even for diffusion velocities several times neoclassical, the errors in the coronal calculations of the total radiation losses in PLT (Princeton Large Torus) are $\leqslant 10-20 \%$. Significant differences of factors of 2 or 3 in the radiated power occur only in a narrow region near the edge of the plasma.

3) TFTR (Tokamak Fusion Test Reactor)

Similar calculations were performed for the inward diffusion of iron in TFTR. Despite the deliberate choice of a diffusion velocity $\sim 4-5$ times neoclassical, the results in Fig. 9 
show that coronal equilibrium is a reasonable approximation for the impurity radiation. For TFTR as well as PLT, the error in the assumption of coronal equilibrium is insignificant compared with the overall uncertainties in the emission rates.

\section{EFFECTS OF IMPURITIES ON FUSION EXPERIMENTS}

Impurities in a D-T fusion plasma dilute the fuel and enhance the radiation losses. The effects of high-z impurities on the Lawson and ignition criteria for reactor experiments have been investigated by Meade [3] using rough estimates for the impurity radiation. We have performed similar calculations with our more detailed emission rates. In addition, we have studied the effects of impurities on the $\mathrm{n}_{\mathrm{e}} \tau$ conditions for a $Q=5$ deuteron beam driven reactor [25].

The power balance equation for a neutral beam injected D-T plasma can be written as:

$$
\frac{3}{2 \tau}\left[n_{e} T_{e}+\sum_{\text {ions }} n_{i} T_{i}\right]+R=0.2\left(P_{F}+P_{B F}\right)+P_{B}
$$

where $\tau$ is the particle energy confinement time; $R$ is the radiation power loss; $\mathrm{P}_{\mathrm{F}}$ is the bulk plasma fusion power from thermal $\mathrm{D}=\mathrm{T}$ reactions; $\mathrm{P}_{\mathrm{BF}}$ is the beam-plasma fusion power; and $\mathrm{P}_{\mathrm{B}}$ is the power of the injected beam. Both $\mathrm{P}_{B F}$ and $\mathrm{P}_{F}$ correspond to the release of $17.6 \mathrm{MeV}$ per reaction. Since only the $3.5-\mathrm{MeV}$ $\alpha$-particles contribute to the plasma energy balance, the total fusion power in Eq. (6) is multiplied by 0.2 . 
The radiation loss term can be written as

$$
\mathrm{R}=\sum_{z} \mathrm{n}_{e^{\mathrm{n}} \mathrm{n}_{z}}+\mathrm{n}_{e^{\mathrm{n}_{o}} \mathrm{~L}_{\mathrm{H}}}
$$

Where the sum is over impurities, $\mathrm{n}_{0}$ is the total hydrogen density, and $\mathrm{L}_{\mathrm{z}}$ and $\mathrm{L}_{\mathrm{H}}$ are the impurity and hydrogen emission rates per ion per free electron.

The fusion power multiplication factor is defined as $Q=$ $\left(\mathrm{P}_{\mathrm{F}}+\mathrm{P}_{\mathrm{BF}}\right) / \mathrm{P}_{\mathrm{B}}$. If we consider a single impurity with density $\mathrm{n}_{z}=\mathrm{fn} \mathrm{n}_{\mathrm{O}}$, then in order to achieve a given $\mathrm{Q}$ in a $\mathrm{n}_{\mathrm{D}}=\mathrm{n}_{\mathrm{T}}=1 / 2 \mathrm{n}_{0}$ plasma it is necessary that:

$$
n_{e} \tau=\frac{3 / 2\left[T_{e}(I+f \bar{Z})+T_{i}(I+f)\right]}{A<\sigma v>E_{F} / 4(I+f \bar{Z})-\left(f L_{z}+L_{H}\right)}
$$

where $\bar{z}$ is the mean $z$ of the impurity, $E_{F}=17.6 \mathrm{MeV}$, and $A=$ $(1+0.2 \mathrm{Q}) /\left[\mathrm{Q}-\left(\mathrm{P}_{\mathrm{BF}} / \mathrm{P}_{\mathrm{B}}\right)\right]$.

For $Q=5$ the total fusion $\alpha$-particle power is equal to the beam power. The $\mathrm{n}_{e} \tau$ requirements for $\mathrm{Q}=5$ experiments were calculated for plasma temperatures of $\mathrm{T}_{\mathrm{e}}=\mathrm{T}_{i}=10$ and $20 \mathrm{keV}$ with various fractions of tungsten, molybdenum, iron and oxygen impurities. The values for $\mathrm{P}_{\mathrm{BF}} / \mathrm{P}_{\mathrm{B}}$ for a 200-keV deuteron beam were taken from Ref. [25]. These results are shown in Fig. 10.

With no bedall injection, the ignition criterion for a contaminated plasma has the same form as Eq. (8) with $A=0.2$. The $\mathrm{n}_{\mathrm{e}}^{\tau}$ conditions for ignition as functions of impurity fraction $f$ are illustrated in Fig. 11.

Finally, the Lawson criterion may be derived from Eq. 
by multiplying the left-hand side by $(1-n)$, where $n$ is the energy recycling efficiency, and by replacing the right-hand side by $\eta P_{T N}$, where $\mathrm{P}_{\mathrm{TN}}$ is the total fusion power released including the bianket reaction [3]. Figure 12 shows the resulting Lawson conditions for a recycyling efficiency $\eta=40 \%$.

It is apparent that impurity radiation can have a devastating effect on fusion reactors. These figures illustrate the impurity control requirements for successful breakeven, $Q=5$, and ignition experiments.

\section{CONCLUSION}

Moderate- and high-z impurity radiation is expected to be significant in both present and future tokamaks. Detailed radiation calculations are required to determine the effects of impurities. Previous order of magnitude estimates appear to be too low.

Because of the large uncertainities in the atomic physics, very accurate calculations are impossible. However, using a simple model with the best available atomic physics we have completed radiation calculations for a variety of tokamak impurities, which should lie within uncertainties of a factor of $2 \rightarrow 4$

Experimental measurements and improved calculations of the atomic physics of high temperature plasmas are vital. When the large uncertainties in the rates are removed, more accurate calculations involving separate ionic species may be performed. 


\section{ACKNOWLEDGMENTS}

The authors are grateful to Dr. Paul Rutherford and Dale Meade of the Princeton Plasma Physics Laboratory for their encouragement and for many useful discussions on the radiation problem in tokamaks. The authors are particularly grateful to Dr. Dale Meade for providing the support for Mr. R. Jensen during the Summer of 1976. The authors also had many useful discussions with E. Hinnov, S. von Goeler, S. Cohen and E. Marmar of the Princeton Plasma Physics Laboratory and J. Scofield of the Lawrence Livermore Laboratory. The second author, D. Post, would like to express his appreciation to Dr. Lowell Wood for his encouragement and support during the course of this calculation. The authors are especially grateful to Miss Lisa Foote for the many hours she spent running the code, analyzing the output and plotting the results.

The authors are grateful to D. Jassby for useful suggestions. 


\section{REFERENCES}

* This work was jointly supported by United States Energy Research and Development Administration Contract E(11-1)3073 and by Lawrence Livermore Laboratory Contract W-7405-ENG-48.

[1] Vershkov, V. A., Mirnov, S. V., Nucl. Fusion 14 (1974) 383 .

[2] TFR Group, Nucl. Fusion 15 (1975) 1053.

[3] Meade, D. M., Nucl. Fusion 14 (1974) 289.

[4] Gibson, A., Nucl. Fusion 16 (1976) 546.

[5] Tarter, B., to appear in JoSRT, 1977.

[6] Katz, I., Private communication.

[7] Merts, A. L., Cowan, R. D., Magee, N. H., Jr. The Calculated Power Output from a Thin Iron-Seeded Plasma LASI Report, LA-6220-MS (1976).

[8] Cox, D. P., Tucker, W. H., Ap. J. 157 (1969) 1157.

[9] Breton, C., De Michelis, C., Matlioli, M., Nucl. Fusion 16 (1976) 891 also EUR-CEA-FC-853 (1976).

[10] Hinnov, E., PPL Report MATT-777 (1970).

[11] Gervids, V. I., Kogan, V. I., JETP Lett. 21 (1975) 150.

[12] Scofield, J., private communication.

[13] Strõmgren, B., Zeit. fũr Ap. $\underline{4}$ (1932) 118.

[14] Chandrasekhar, S., An Introduction to the Study of Stellar Structure, Chicago (1939) Chap. VII.

[15] Jordan, C., Mon. Not. R. Astron. Soc. 142 (1969) 501. 
[16] Post, D., Jensen, R., Tarter, B., Grasberger, W., and Lokke, W. PPPL Report (to appear)..

[17] Bates, D. R., Kingston, A. E., McWhirter, R. W. P. Proc. Roy. Soc. A267 (1962) 297.

[18] Burgess, A., Ap. J. 141 (1965) 1588.

[19] Burgess, A., Proceedings of the Second Harvard-Smithsonian Conference on Stellar Atmospheres, (1965) p. 47.

[20] Seaton, M., Mon. Not. R. Astron. Soc. 119 (1959) 81.

[21]. Bethe, H., Salpeter, E., Quantum Mechanics of One and Two Electron Atoms, New York (1957). Chap. IV.

[22] Karzas, W. J., Latter, R., Ap. J. Suppl. 6 (1961) 167.

[23] Cohen, S., Marmar, E., Cecchi, J., Phys. Rev. Lett. 35 (1975) 1507 also PPPL Report 1248 (1976).

[24] Rutherford, P. H., private communication.

[25] Jassby, D. L., Princeton Plasma Physics Laboratory PPPL-1280 (1976). 
T A B L E 1

Ion Species

$\mathrm{XXI}$

XXII

XXIII

XXIV

XXV

Avcrage

Ion Model

0.0404

0.0850

0.1505

0.2540

0.4467

Merts,
et.al: [7] . 0.020

0.056

0.131

0.261

0.525 


\section{TABLE 2}

ATC

$1.4\left(1-\left(\frac{r}{a}\right)^{2}\right)^{3}$

Temperature（KEV)

$\begin{array}{ll}i & \text { ION } \\ \stackrel{1}{T} & \text { TEMPERATURE (KEV) }\end{array}$

\section{ELECTRON}

DENSITY

DIFFUSION

VELOCITIES (CM/MS)
$2.5 \times 10^{13}\left(1-\left(\frac{r}{a}\right)^{2}\right)$

$$
2.1-0.21 \mathrm{x}
$$

( $\mathrm{x}$ is penetration
distance)
PLT

$2 \cdot\left(1-\left(\frac{r}{a}\right)^{2}\right)$

$10 \cdot\left(1-\left(\frac{r}{a}\right)^{2}\right)$

$1 \cdot\left(1-\left(\frac{r}{a}\right)^{2}\right)$

$\left.5 \cdot\left(1-i \frac{r}{a}\right)^{2}\right)$

$5 \times 10^{13}\left(1-\left(\frac{r}{2}\right)^{3}\right)$

$5 \times 10^{13}\left(1-\left(\frac{r}{a}\right)^{3}\right)$

TFTR

$$
0.5,2 \text {. }
$$

2. 


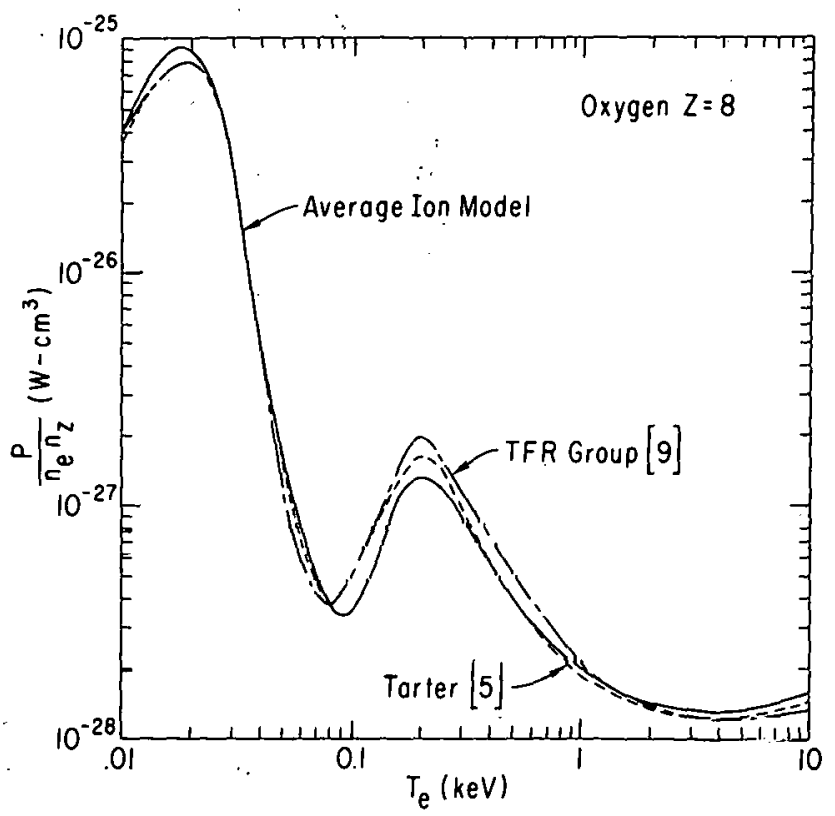

772074

Fig. 1 Total radiated power as a function of electron temperature for oxygen.

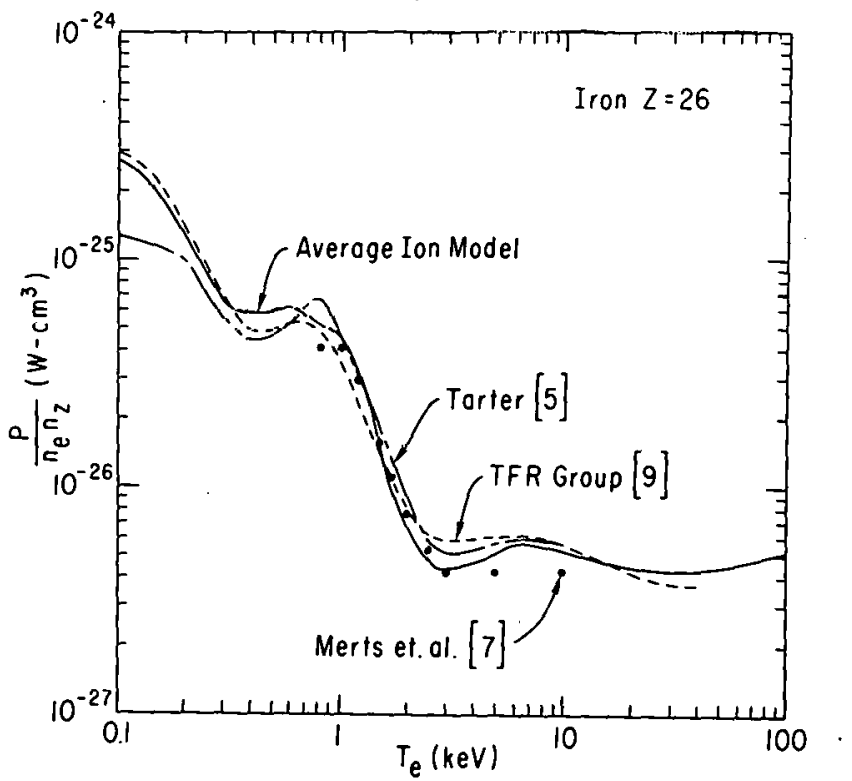

772069

Fig. 2 Total radiated power as a function of electron temperature for iron. 


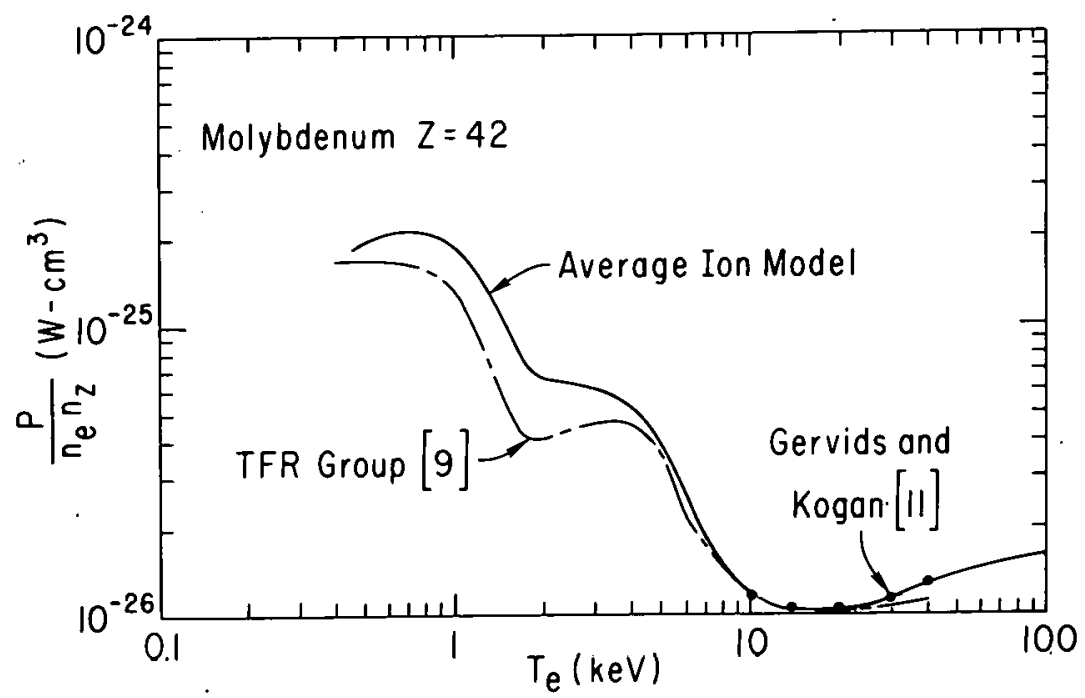

772068

Fig. 3 Total radiated power as a function of electron temperature for molybdenum.

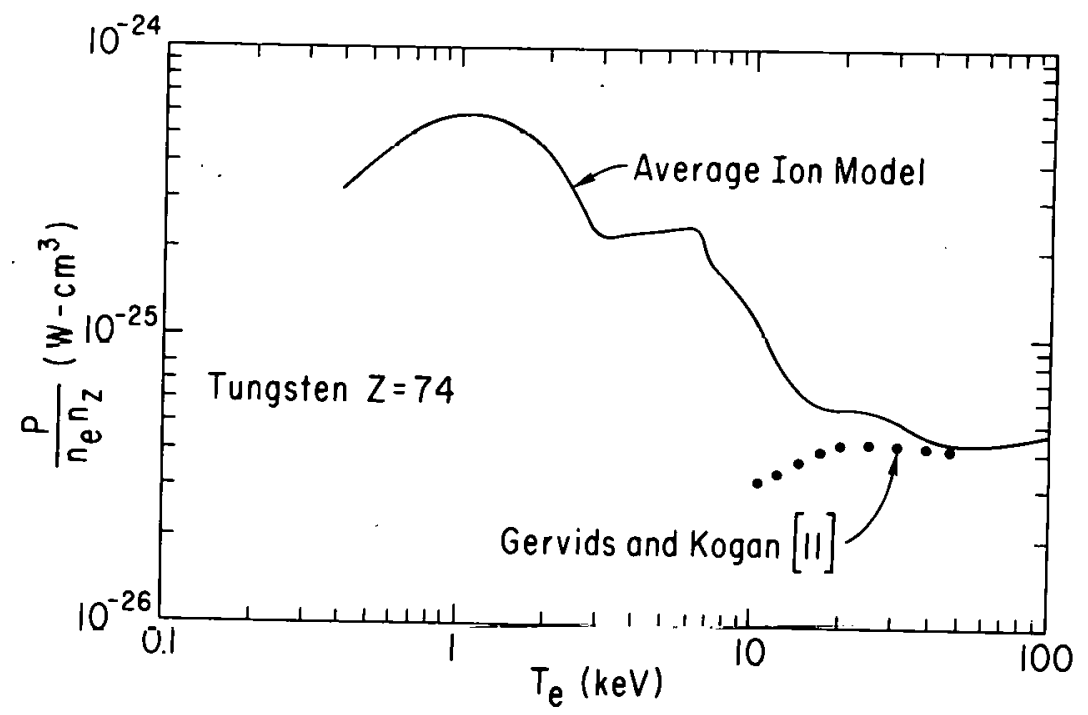

772067

Fig. 4 Total radiated power as a function of electron temperature for tungsten. 


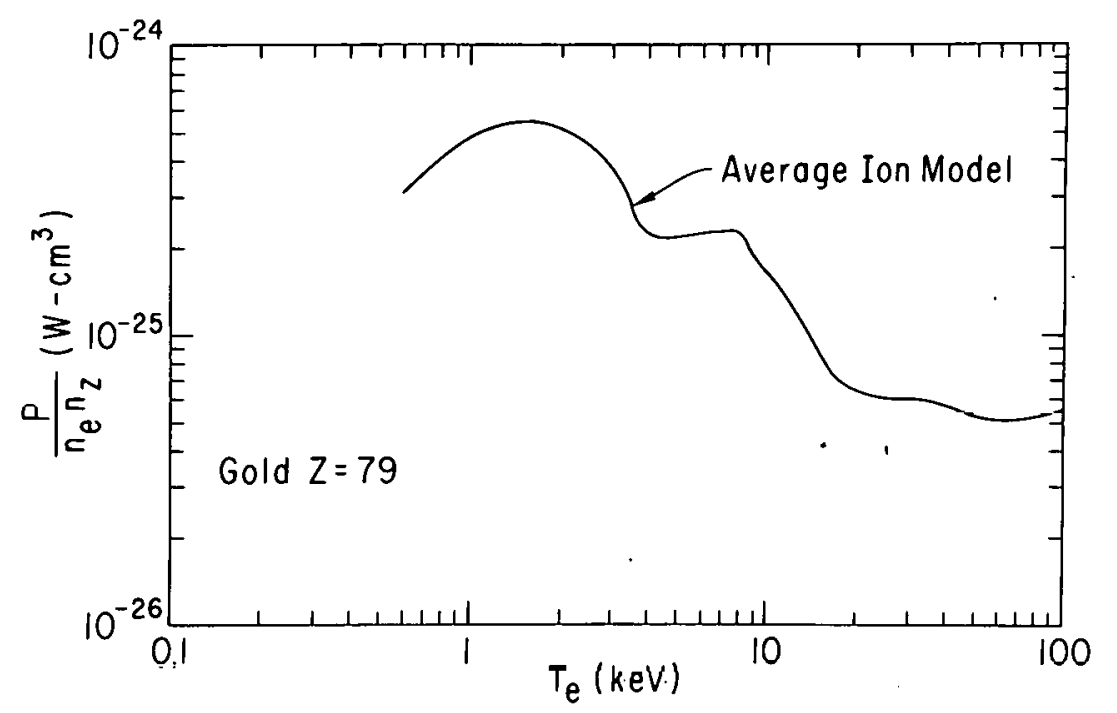

772066

Fig. 5 Total radiated power as a function of electron temperature for gold.

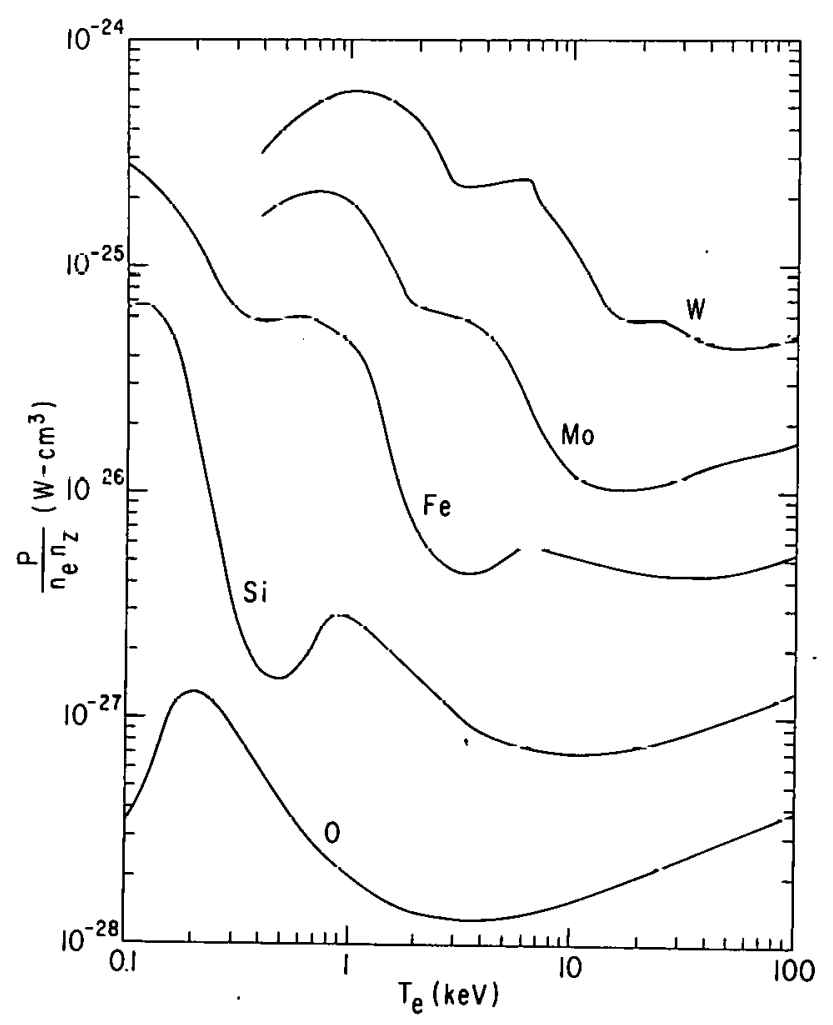

772071

Fig. 6 Comparison of total radiated power for a variety of impurities showing the increase with $z$. 


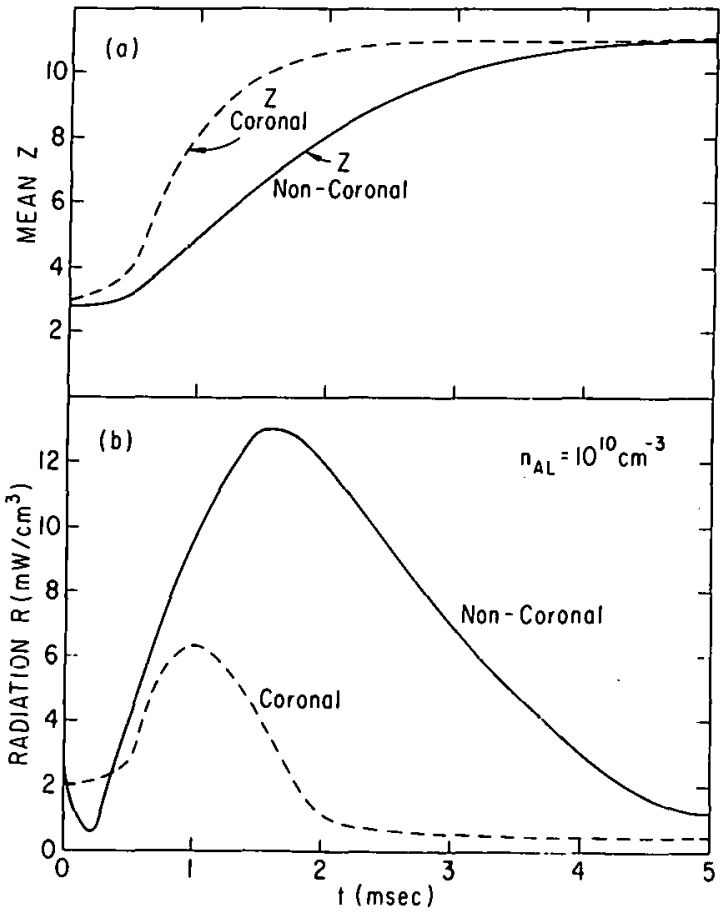

Fig. 7 Aluminum injected in ATC:

(a) Radial $\bar{z}$ profile for coronal and non-coronal calculations.

(b) Coronal and non-coronal radiated power $\mathrm{R}$. 


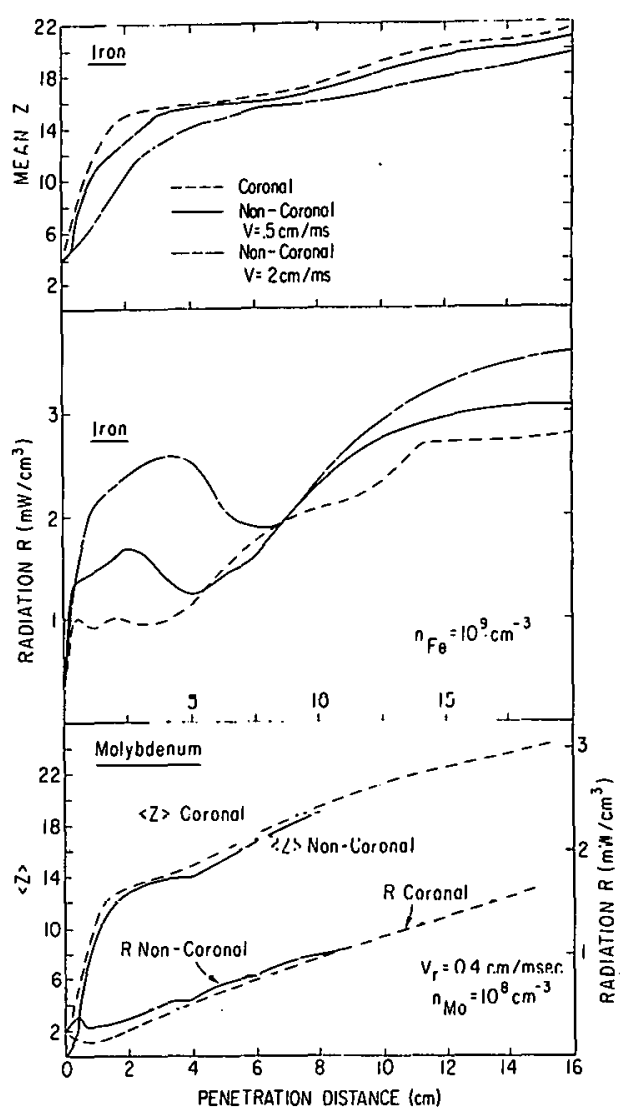

772075

Fig. 8 Impurity diffusion in PLT:

(a) Coronal and non-coronal calculations of $\bar{z}$ for iron with $\mathrm{v}=0.5$ and $2 . \mathrm{cm} / \mathrm{ms}$.

(b) Coronal and non-coronal calculations of the total radiated power $\mathrm{R}$ for iron.

(c) Coronal and non-coronal results for $\bar{z}$ and radiated power $\mathrm{R}$ for molybdenum with $\mathrm{v}=0.4 \mathrm{~cm} / \mathrm{ms}$. 


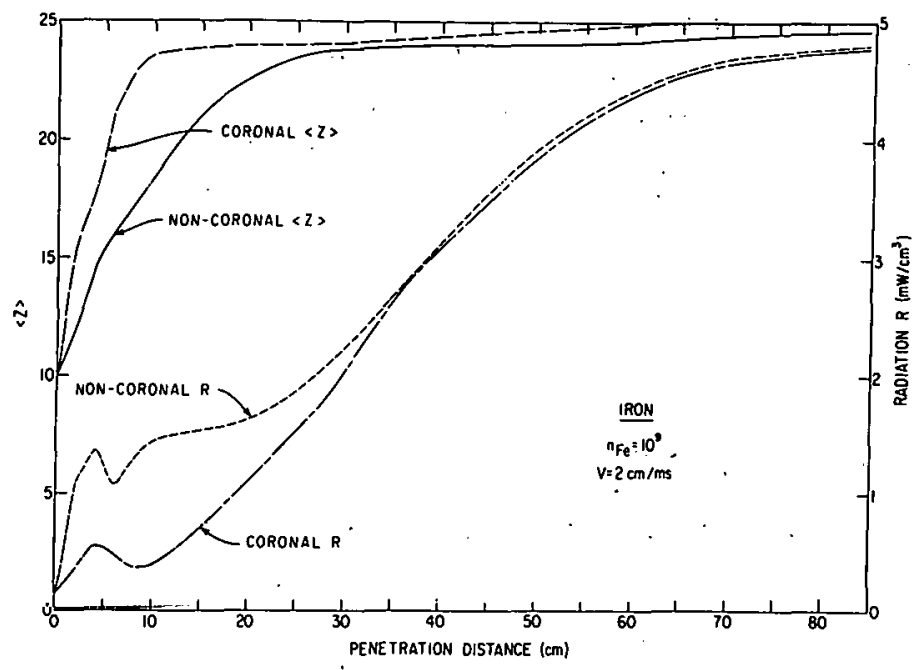

772100

Fig. 9 Coronal and non-coronal calculations of $\bar{z}$ and total radiated power $\mathrm{R}$ for iron diffusing inward in TFTR with $\mathrm{v}=2$. $\mathrm{cm} / \mathrm{ms}$.

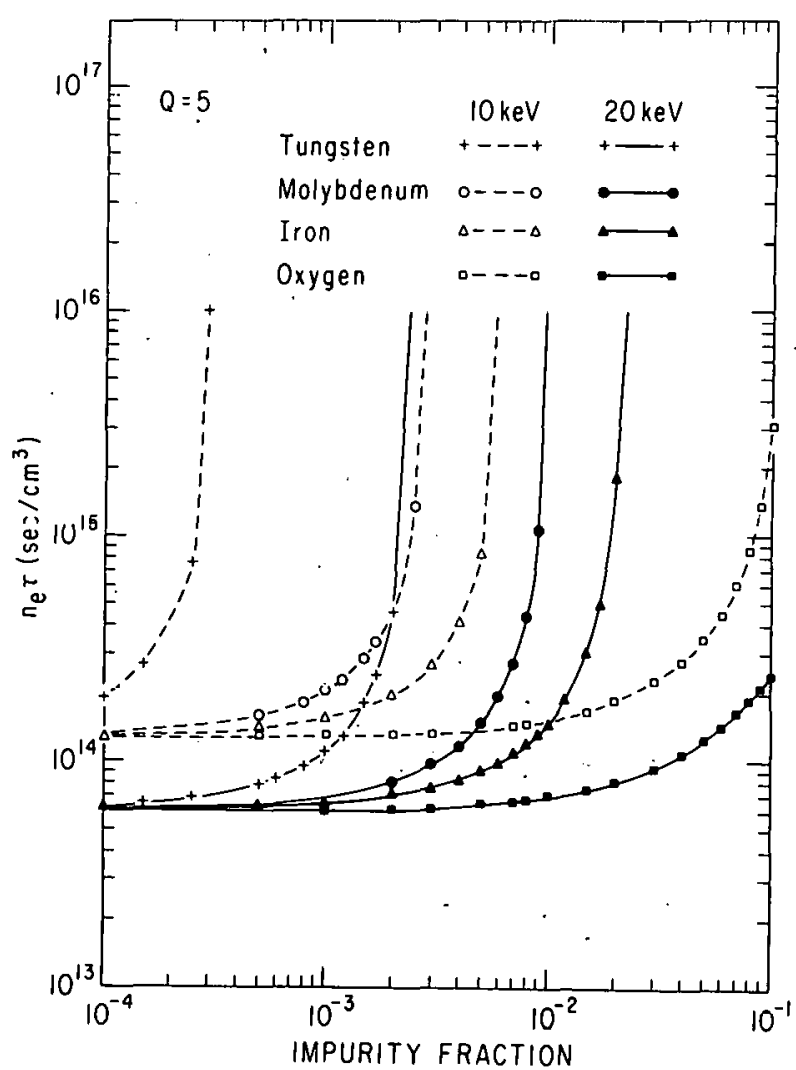

772073

Fig. $10, n_{e}^{\tau}$ requirements as functions of impurity fraction $f$ for $Q=5$ beam driven $D-T$ plasmas at temperatures of 10 and $20 \mathrm{keV}$. 